\section{Recht und Bioethik. Verweisungszusammenhänge bei der Normierung der Lebenswissenschaften.}

\author{
Von Tade Matthias Spranger. (Jus Publicum. Beiträge zum \\ Öffentlichen Recht, Bd. 190), Verlag Mohr Siebeck, Tübin- \\ gen 2010, XVI u. 445 S., geb., $€ 114,00$
}

Zu den „life sciences“ gibt es eine kaum noch zu überschauende Fülle an nationalen, supranationalen und internationalen Vorschlägen und Maßgaben, an empfehlenden Berichten, Stellungnahmen oder Deklarationen, an völker- und europarechtlichen Anforderungen, die alle mehr oder weniger die für Rechtssetzung und Rechtsanwendung Verantwortlichen herausfordern. Die Bonner Habilitationsschrift erörtert die ebenso aktuelle wie komplexe Frage, welche Funktion bioethischen Entwürfen im Kontext positiver Normierung der Lebenswissenschaften zukommt. Vornehmlich die englischsprachige, auch die deutsche Literatur dazu ist überaus reichhaltig. Mochten darum die Schriften des Rezensenten ungenannt bleiben, so durften doch wegweisende Publikationen im Literaturverzeichnis nicht fehlen: Häberles „Das Menschenbild im Verfassungsstaat“ etwa oder Böckenfördes Aufsätze: „Menschenwürde als normatives Prinzip“ (JZ 2003, 809) und „Die Würde des Menschen war unantastbar“ (FAZ Nr. 204 v. 3.9.2003, S. 33), die sich kritisch auch mit der Neukommentierung des Art. 1 GG aus der Feder Herdegens, des akademischen Lehrers unseres Autors, auseinandersetzen. Die Leser dieser Zeitschrift werden den grundlegenden Beitrag zum Recht der Biomedizin von Zuck vermissen, der dieses Feld gekennzeichnet sieht durch Institutionalisierung, Integration, Internationalisierung und Instabilität (MedR 2008, 57).

Die neuen naturwissenschaftlich-technischen Machbarkeiten in der Medizin werfen Grundfragen des menschlichen Lebens auf, die über die berufsständisch geprägte Arztethik hinausführen zur Bioethik. „Bioethik ist damit zunächst eine um den kulturellen Aspekt angereicherte Medizinethik" (S. 28). Sie befasst sich mit der biowissenschaftlich ausgebildeten Human-, insbesondere der Fortpflanzungs- und Genmedizin. Soweit bioethische Themen in das Recht einschlagen, identifiziert die Jurisprudenz sie als spezifisch juristische Probleme, entdeckt und behandelt sie als solche. ,,Ohne ihren interdisziplinären Charakter einzubüßen, gewinnt die bioethische Debatte so aller Auseinandersetzungen zum Trotz an praktischem Bezug und Objektivität, zumal die Setzung allgemeinverbindlicher Standards ausschließlich in den Formen des positiven Rechts erfolgen kann“ (S. 59). Ethische Maßgaben, so arbeitet der Autor heraus, ,,verdichten sich nur dann zu Rechtssätzen, wenn sie unter Beachtung der formellen und materiellen Anforderungen in deren konkrete Form gegossen werden“. Es gilt, was Altmeister Wieacker bereits gelehrt hat: Legitimer Schauplatz für den Kampf um die Einfügung neuer materialer Ethik in die Rechtsordnung ist der Gesetzgebungsprozeß (S. 409).

Kulturelle Verschiedenheiten, das Ineinandergreifen philosophischer, religiöser, weltanschaulicher Ideen, die Frage des institutionellen Rahmens, das Tempo der naturwissenschaftlichen Fortschritte, die Sorge vor drohender Absenkung rechtsstaatlich verbürgter Gewährleistungen erschweren Kompromisse und das Festlegen von Mindeststandards im Wege einer Internationalisierung der Bioethik. Dennoch geht ein Trend dahin.

Im umfänglichsten Kapitel „Völkerrecht“ untersucht der Autor exemplarisch bioethische Dokumente internationaler Organisationen: Das Biomedizinübereinkommen des Europarates von 1997, das Klonprotokoll des Europarates von 1998, die UNESCO-Erklärung zum Humangenom von 1997, die UNESCO-Erklärung zu humangenetischen Daten aus dem Jahre 2003, die Allgemeine Erklärung über Bioethik und Menschenrechte der UNESCO-Generalkonferenz von 2005, die 1993 in Kraft getretene Konvention über die biologische Vielfalt und ferner das damit in Zusammenhang stehende Biosafety Protokoll, das 135 Staaten im Jahr 2000 annahmen, schließlich das Agreement on Trade-Related Aspects of Intellectual Property Rights (TRIPS) im Dienste der medizinischen Versorgung in Entwicklungsländern.

Das folgende Kapitel gilt dem Europäischen Recht. Die Existenz des Patentrechts hält der Autor mit Grund für ethisch gerechtfertigt.

Prof. Dr. iur. Dr. h.c. Adolf Laufs,

Heidelberg, Deutschland
Reformbedarf erkennt er „im Hinblick auf den Schutzumfang sogenannter Stoffpatente im Bereich biotechnologischer Erfindungen“" (S. 247). Nicht nur im Lebensmittelrecht, auch in der EG-Freisetzungsrichtlinie von 2001 gewinnen ethische Gesichtspunkte Raum. Die Charta der Grundrechte der Europäischen Union aus dem Jahr 2000 folgt weitgehend den Kriterien und Grundsätzen des Biomedizinübereinkommens des Europarates. Der Autor sieht in seiner Gesamtbewertung die europäische Gesetzgebung gekennzeichnet „durch ein hohes Maß an Skepsis gegenüber den Entwicklungen der modernen Biomedizin und Gentechnologie“ und eine „Verengung der Handlungsspielräume“ (S. 275). „Wissenschaftlich nicht belegbare Gesichtspunkte“ hätten in der EU ,,ein weitaus größeres Gewicht ... als ... in anderen Teilen der Welt" (S. 276).

Im Kapitel „Exemplarische nationale Regulierungen“ erscheint Deutschland mit Debattenbeiträgen vorwiegend positivrechtlicher Art zur Forschung mit Nichteinwilligungsfähigen, zur Präimplantationsdiagnostik (mit problematischer Analogie zu Abtreibungskriterien) und zum Stammzellgesetz. Es folgt eine Analyse der Bioethikgesetze im verfassungsrechtlichen Kontext Frankreichs, deren Regulierungsdichte wesentlich auf das subjektiv-rechtliche Verständnis des Zugangs zu medizinischen Versorgungseinrichtungen zurückgeht. Den dritten Teil der nicht näher begründeten Auswahl bildet Australien.

Das folgende Kapitel bietet lehrreiche kritische Besprechungen von Richtersprüchen: der Entscheidung des EGMR zum Schutz des ungeborenen Lebens (NJW 2005, 727), deren Zurückhaltung der Autor zu Recht kritisiert, der Entscheidung des EuGH zur Biopatentrichtlinie, des Österreichischen Verfassungsgerichtshofes zum Fortpflanzungsmedizingesetz, außerdem der Rechtsprechung des Schweizerischen Bundesgerichts, der Entscheidung des BSG zur Überkreuzlebendnierenspende und des Bundespatentgerichts zur Nutzung embryonaler Stammzellen.

$\mathrm{Zu}$ den Erträgen des stoffgesättigten, durch Zusammenfassungen gut aufnehmbaren Werkes gehört die Einsicht in die Offenheit ethischer Maximen und damit die „Gefährdung der Rechtssicherheit durch Inkorporation ethischer Prinzipien in positivrechliche Normen“, auch die Erkenntnis der „Gefahr einer Instrumentalisierung der Bioethik durch die Rechtspolitik“" (S. 411). Der Begriff des Biorechts biete, so der überzeugende Schluss, „die Möglichkeit, wieder verstärkt auf den Charakter einer Analyse als rein gesetzesrechtliche hinzuweisen". Bioethik und Biorecht bleiben freilich aufeinander angewiesen, auch bei formeller und substantieller Trennung. Am Ende des Studiums einer so reichhaltigen wie uneinheitlichen Materie bleibt Ratlosigkeit angesichts der Mannigfaltigkeit und Widersprüchlichkeit der Regeln - ohne System und ohne allgemeine feste Ausgangspunkte: Verworrenheiten in der westlichen Welt.

\section{DOI: 10.1007/s00350-011-2966-4}

\section{Jahrbuch für Wissenschaft und Ethik, Bd. 15.}

Herausgegeben von Ludger Honnefelder und Dieter Sturma. Verlag De Gruyter, Berlin 2010, 496 S., kart., € 59,95

Diese knappe Anzeige will den jüngsten, überaus gehaltvollen Band empfehlen, den wir wieder dem Institut für Wissenschaft und Ethik (IWE) verdanken, das inzwischen eine zentrale wissenschaftliche Einrichtung der Universität Bonn geworden ist. Das Jahrbuch bietet in der ersten Hälfte seines Umfangs zwölf Abhandlungen. Davon befassen sich sechs in dem von Jan P. Beckmann gestalteten Schwerpunkt mit der rechtlichen, medizinischen und ethischen Beurteilung des Patientenverfügungsgesetzes von 2009 und der Patientenverfügung (S. 139-241). Dabei legen die Juristen Klaus Kutzer und Jochen Taupitz eindrucksvoll die offen gebliebenen Probleme dar. Der Dokumentationsteil des Bandes enthält Empfehlungen und Stellungnahmen gewichtiger nationaler und internationaler Organisationen teils in deutscher, teils in englischer Sprache zu unterschiedlichen wissenschaftsethischen Hauptfragen, unter anderen auch zur aktuellen Demenz- und Patientenverfügungs-Thematik.

Prof. Dr. iur. Dr. h.c. Adolf Laufs,

Heidelberg, Deutschland 